\title{
Cosmological constraints on holographic dark energy models under the energy conditions
}

\author{
Ming-Jian Zhang ${ }^{1}$, Cong $\mathrm{Ma}^{1}$, Zhi-Song Zhang ${ }^{2}$, Zhong-Xu Zhai ${ }^{3}$, Tong-Jie Zhang, ${ }^{1,4}$ \\ ${ }^{1}$ Department of Astronomy, Beijing Normal University, Beijing, 100875, China \\ ${ }^{2}$ Department of Aerospace Engineering, School of Astronautics, \\ Harbin Institute of Technology (HIT), Harbin Heilongjiang, 150001, China \\ ${ }^{3}$ Department of Physics, Institute of Theoretical Physics, \\ Beijing Normal University, Beijing, 100875, China and \\ ${ }^{4}$ Center for High Energy Physics, Peking University, Beijing, 100871, China
}

\begin{abstract}
We study the holographic and agegraphic dark energy models without interaction using the latest observational Hubble parameter data (OHD), the Union2.1 compilation of type Ia supernovae (SNIa), and the energy conditions. Scenarios of dark energy are distinguished by the cut-off of cosmic age, conformal time, and event horizon. The best-fit value of matter density for the three scenarios almost steadily located at $\Omega_{m 0}=0.26$ by the joint constraint. For the agegraphic models, they can be recovered to the standard cosmological model when the constant $c$ which presents the fraction of dark energy approaches to infinity. Absence of upper limit of $c$ by the joint constraint demonstrates the recovery possibility. Using the fitted result, we also reconstruct the current equation of state of dark energy at different scenarios, respectively. Employing the model criteria $\chi_{\min }^{2} / d o f$, we find that conformal time model is the worst, but they can not be distinguished clearly. Comparing with the observational constraints, we find that SEC is fulfilled at redshift $0.2 \lesssim z \lesssim 0.3$ with $1 \sigma$ confidence level. We also find that NEC gives a meaningful constraint for the event horizon cut-off model, especially compared with OHD only. We note that the energy condition maybe could play an important role in the interacting models because of different degeneracy between $\Omega_{m}$ and constant $c$.
\end{abstract}

PACS numbers: $98.80 .-\mathrm{k}, 95.36 .+\mathrm{x}$

\section{INTRODUCTION}

Several independent cosmological observations probed a phenomena of an accelerated expansion of the universe. Examples include the Type Ia supernovae (SNIa) observations [1], large scale structure [2], and cosmic microwave background (CMB) anisotropies [3]. Generally, dark energy models or some modified gravity are approved to be theoretical explanations of this acceleration. For the dark energy, however, many variants are listed as one candidate. They are usually in the form of cosmological constant, quintessence [4], Kessence [5], tachyon [6], phantom [7], ghost condensate [8], quintom [9] and the holographic dark energy which will be investigated in this paper. Observationally, many of them can fit well with current observations.

Historically, the holographic dark energy is addressed to alleviate or remove the cosmological constant problems [4, 10, 11]. From the origin, it dates from the holographic principle and inspire from the Bekenstein entropy bound. In an effective quantum field theory, the total entropy in a box of size $L$ with UV cut-off $\Lambda$ relating to the quantum zero-point energy [12, 13] should satisfy the relation $L^{3} \Lambda^{3} \leqslant S_{B H} \equiv \pi M_{p}^{2} L^{2}$, where $S_{B H}$ is the entropy of a black hole within the same size as $L$, and $M_{p} \equiv 1 / \sqrt{8 \pi G}$ is the reduced Planck mass. Cohen et al. [14] suggested that a short distance cut-off in quantum field theory is related to a long distance cut-off, due to the limit set by formation of a black hole. If $\rho_{\Lambda}$ is the quantum zero-point energy density caused by a short distance cut-off, the total energy in a region of size $L$ should not exceed the

*Electronic address: tjzhang@bnu.edu.cn mass of a black hole of the same size, namely, $L^{3} \rho_{\Lambda} \leqslant L M_{p}^{2}$. The largest $L$ allowed is the one saturating this inequality. The energy density should, therefore, satisfy

$$
\rho_{\Lambda}=3 c^{2} M_{p}^{2} L^{-2},
$$

where $c$ is a dimensionless constant introduced for convenience, but indicating the abundance of matter or dark energy component. It should not be confused with the speed of light $c$ (light). If considering the cut-off $L$ on the cosmology as the size of the universe, the holographic dark energy finally was born. Initially, the cut-off $L$ is construed as the Hubble horzion $H^{-1}$ [15-17]. However, it can not deduce the accelerating expansion of the universe [17]. Subsequently, some length scales are proposed, such as the particle horizon, future event horizon. This kind of models are usually named the holographic dark energy. Conversely, some agegraphic dark energy models corresponding to temporal scales also come into being in following years, e. g. the age of the universe or the conformal time. Nevertheless, the latter is also accused of classically unstable and worse fitting result [18]. This is mainly because the constant $c$ is difficult to determinate in such models. On the other hand, instead of the $\rho_{\Lambda}$ as the total energy, many modified dark energy models are proposed [19].

The confrontation of theories and data, however, is not all one may perform in the face of the proliferation of dark energy proposals. One of the many interesting approaches makes use of the so-called energy conditions. These conditions were introduced by Hawking et al. [20] as coordinate-invariant inequality constraints on the energy-momentum tensor that appears in the Einstein field equation. Due to their simplicity and model independence, the energy conditions are frequently discussed in the general context of gravitation [21]. Of the many proposed energy conditions, the ones we employ 
in this paper are the null, weak, strong, and dominant energy conditions (abbreviated respectively as NEC, WEC, SEC, and DEC). The energy conditions in the setting of a homogeneous and isotropic Friedmann-Robertson-Walker (FRW) universe summarized by Carroll [22, Chapter 4] can be expressed in terms of the energy density $\rho$ and the pressure $p$ as following:

$$
\begin{array}{lll}
\text { NEC : } & \rho+p \geqslant 0, \\
\text { WEC : } & \rho \geqslant 0 \quad \text { and } \quad \rho+p \geqslant 0, \\
\text { SEC : } & \rho+3 p \geqslant 0 \quad \text { and } \quad \rho+p \geqslant 0, \\
\text { DEC : } & \rho \geqslant 0 \quad \text { and }-\rho \leqslant p \leqslant \rho .
\end{array}
$$

The energy conditions has been useful in discussing the general property of fluid models [23, and references therein]. The energy conditions were applied to cosmology by Visser [24-26]. It has been shown that constraints on a variety of cosmological variables or parameters can be predicted from the energy conditions, such as the Hubble parameter, luminosity and angular diameter distances, lookback time [25], total density parameter $\Omega(z)$, energy density $\rho(z)$, and pressure $p(z)$ [27]. In a word, the energy conditions have been an useful tool for our understanding of the Universe's evolution.

Unlike first-principle laws of physics, the energy conditions are not expected to hold a priori, nor are they found to do so from data. It has been shown that the WEC and DEC are fulfilled for $z \leq 1$ and $z \lesssim 0.8$ by supernova data with $3 \sigma$ confidence levels, respectively [28]. Nevertheless, SEC violation is a typical trait of a positive cosmological constant $\Lambda$ [29] and other dark energy models [30]. It is also said that the WEC violate the quantum field theory due to arbitrarily negative renormalized energy density may occur at some points of spacetime [31]. If extended regions of large negative energy density emerge in the nature, exotic and possibly undesirable phenomena may be allowed, ranging from violations of the second law of thermodynamics and cosmic censorship to the creation of time machines and warp drives [32].

Recently, Wu et al. [23] studied the likelihood of energy condition violations in the history of the universe. They found that the data suggest a fulfillment of null and dominant energy conditions, but a violation of strong energy condition, especially for low redshift $(z \leqslant 0.3)$. They also noted the difficulty of assessing the possibility of SEC violation at the high redshift. Moreover, their result for SEC hints at a recent transition from deceleration to acceleration with the transition redshift $z \approx 0.5$ under the ignorance of bias at the high redshift. Therefore, the SEC fulfillment appears disfavored as a test for cosmological models. On the contrary, a dark energy model is expected to reproduce its violation for recent eras of cosmic evolution.

This paper is organized as follows: In Sec. II the basic expansion rate for general holographic and agegraphic dark energy models (collectively abbreviated as HDE) is derived and calculated for different choices of the IR cut-off. Proceeding to Sec. III we subject the models to our data constraints and energy condition analyses. Our main results and discussions are presented in Sec. IV

\section{HOLOGRAPHIC AND AGEGRAPHIC DARK ENERGY MODELS}

In this section we consider the HDE models without interaction [33] between the dark energy and matter (including both baryon matter and cold dark matter). The Friedmann equation for a spatially flat FRW model ignoring the radiation reads [34, 35]

$$
\rho_{m}+\rho_{\Lambda}=3 M_{p}^{2} H^{2}
$$

By introducing $\Omega_{m}=\rho_{m} /\left(3 M_{p}^{2} H^{2}\right)$ and $\Omega_{\Lambda}=\rho_{\Lambda} /\left(3 M_{p}^{2} H^{2}\right)$, the Friedmann equation can also be cast as $\Omega_{m}+\Omega_{\Lambda}=1$. With the dark energy pressure $p=\omega \rho$ and cold dark matter pressure $p=0$, the total pressure $p$ is given by

$$
p=3 M_{p}^{2} \omega c^{2} L^{-2},
$$

where $\omega$ is called the equations of state $(\mathrm{EoS})$ parameter. Following previous works [34, 36-38], the continuity equations for dark energy and matter respectively are

$$
\dot{\rho}_{m}+3 H\left(1+\omega_{m}\right) \rho_{m}=0, \quad \dot{\rho}_{\Lambda}+3 H\left(1+\omega_{\Lambda}\right) \rho_{\Lambda}=0,
$$

where a dot above denotes the derivative with respect to the cosmic time $t$. Connecting Eqs. (3) and (5) we can obtain

$$
2 M_{p}^{2} \dot{H}+3 M_{p}^{2} H^{2}+\omega_{m} \rho_{m}+\omega_{\Lambda} \rho_{\Lambda}=0 .
$$

By virtue of $\omega_{m}=0$, the EoS of HDE, with the help of the second equation of Eq. (5), can be expressed as

$$
\begin{aligned}
\omega_{\Lambda} & =-1-\frac{1}{3 H} \frac{\dot{\rho}_{\Lambda}}{\rho_{\Lambda}}=-1-\frac{1}{3 H \Omega_{\Lambda}}\left(\frac{2 \Omega_{\Lambda}}{H} \frac{d H}{d t}+\frac{d \Omega_{\Lambda}}{d t}\right) \\
& =-1-\frac{2}{3}\left(\frac{d \ln H}{d \ln a}+\frac{1}{2} \frac{d \ln \Omega_{\Lambda}}{d \ln a}\right) .
\end{aligned}
$$

Applying the EoS of HDE, Eq. (6) reduces to

$$
\frac{d \ln H}{d \ln a}-\frac{1}{2} \frac{\Omega_{\Lambda}}{\left(1-\Omega_{\Lambda}\right)} \frac{d \ln \Omega_{\Lambda}}{d \ln a}+\frac{3}{2}=0 .
$$

Meanwhile, we get the Hubble parameter $H(z)=H_{0} E(z)$ with Eq. (7) and Friedmann equation Eq. (3), where the expansion rate $E(z)$ is given by [37]

$$
E^{2}(z)=\Omega_{m 0}(1+z)^{3}+\Omega_{\Lambda 0} \exp \left[3 \int_{0}^{z} \frac{1+\omega_{\Lambda}\left(z^{\prime}\right)}{1+z^{\prime}} d z^{\prime}\right] .
$$

Here the subscript " 0 " denotes the present value of a quantity.

To further quantify a model, a choice of the IR cut-off is needed. Scenarios summarized by Chen et al. [38] contains the Hubble horizon, the particle horizon, the event horizon, the age of the universe and the conformal time [37, 39]. However, an accelerating expansion of the universe cannot be achieved when the Hubble horizon or the particle horizon is chosen as the IR cut-off. In this paper, we mainly aim at the last three scenarios. Recently, Zhai et al. [37] investigated four of them and found that the event horizon is more preferable than Hubble horizon scenario. However, both two temporal scenarios better recovers the $\Lambda \mathrm{CDM}$ model than the spatial scenarios. In our following calculations we choose the natural units of the speed of light $c$ (light) $=1$. Our final results will be presented in dimensionless quantities. 


\section{A. Cosmic age cut-off}

The first scenario, the cosmic age cut-off, is defined as

$$
t_{\Lambda}=\int_{0}^{t} d t^{\prime}=\int_{0}^{a} \frac{d a^{\prime}}{H a^{\prime}}
$$

In this case, the age of universe is considered as a time scale. The corresponding spatial scale is obtained after multiplication by the speed of light $c$ (light). According to Eq. (1) the resulting dark energy density is

$$
\rho_{\Lambda}=3 c^{2} M_{p}^{2} t_{\Lambda}^{-2}
$$

With the dark energy density parameter $\Omega_{\Lambda}=\rho_{\Lambda} /\left(3 M_{p}^{2} H^{2}\right)$ and the definition Eq. (10), we find

$$
\int_{0}^{a} \frac{d \ln a^{\prime}}{H}=\frac{c}{H} \sqrt{\frac{1}{\Omega_{\Lambda}}} .
$$

Taking the derivative with respect to $\ln a$, a differential equation is derived

$$
\frac{d \ln H}{d \ln a}+\frac{1}{2} \frac{d \ln \Omega_{\Lambda}}{d \ln a}+\frac{\sqrt{\Omega_{\Lambda}}}{c}=0 .
$$

Then, from Eqs. (8) and (13), the evolution of $\Omega_{\Lambda}$ can be found to satisfy

$$
\frac{d \Omega_{\Lambda}}{d z}=-2 \Omega_{\Lambda}\left(1-\Omega_{\Lambda}\right)\left(\frac{3}{2}-\frac{\sqrt{\Omega_{\Lambda}}}{c}\right)(1+z)^{-1} .
$$

Meanwhile, the EoS for HDE can be obtained from Eqs. (7) and (13)

$$
\omega_{\Lambda}=\frac{2}{3 c} \sqrt{\Omega_{\Lambda}}-1
$$

Accelerated expansion requires $c>\sqrt{\Omega_{\Lambda}}$ in order to satisfy $\omega<-1 / 3$. When the constant $c$ approaches infinity, the $\Lambda C D M$ model is recovered. The expansion rate under this scenario can eventually be determined by Eqs. (9) and (15).

\section{B. Conformal time cut-off}

The second scenario we consider is the conformal time as the IR cut-off. It is in the form

$$
\eta_{\Lambda}=\int_{0}^{t} \frac{d t^{\prime}}{a}=\int_{0}^{a} \frac{d a^{\prime}}{H a^{2}},
$$

which is the total comoving distance that light could travel [40]. In this case, the conformal time is considered as a temporal scale, and we can again convert it to a spatial scale to be used as the IR cut-off $L$. Analogous to Section $\Pi$ A we obtain a differential expression of $\Omega_{\Lambda}$ with respect to $\ln a$ :

$$
\frac{d \ln H}{d \ln a}+\frac{1}{2} \frac{d \ln \Omega_{\Lambda}}{d \ln a}+\frac{\sqrt{\Omega_{\Lambda}}}{a c}=0 .
$$

We can go further with the aid of Eq. (8):

$$
\frac{d \Omega_{\Lambda}}{d z}=-2 \Omega_{\Lambda}\left(1-\Omega_{\Lambda}\right)\left[\frac{3}{2}(1+z)^{-1}-\frac{\sqrt{\Omega_{\Lambda}}}{c}\right] .
$$

The EoS for HDE can be obtained from Eqs. (7) and (17)

$$
\omega_{\Lambda}=\frac{2}{3} \frac{\sqrt{\Omega_{\Lambda}}}{c}(1+z)-1
$$

which corresponds to an acceleration when $c>\sqrt{\Omega_{\Lambda}}(1+z)$. With $c \rightarrow \infty$ it will also recover the $\Lambda \mathrm{CDM}$ model. The corresponding expansion rate Eq. (9) of this scenario can also be solved.

\section{Event horizon cut-off} by

For our last model, we consider the event horizon [41] given

$$
R_{E}=a \int_{t}^{\infty} \frac{d t^{\prime}}{a\left(t^{\prime}\right)}=a \int_{a}^{\infty} \frac{d a^{\prime}}{H a^{\prime 2}},
$$

which is the boundary of the volume a fixed observer may eventually observe. We now identify this scale as the cut-off length $L$ as it appears in Eq. (1). With the introduction of dark energy density parameter $\Omega_{\Lambda}$, and performing the same analysis, we obtain the relation from Eq. (20)

$$
\int_{a}^{\infty} \frac{d \ln a^{\prime}}{H a^{\prime}}=\frac{c}{H a} \sqrt{\frac{1}{\Omega_{\Lambda}}} .
$$

Taking the derivative with respect to $\ln a$ of Eq. (21), we get a differential equation

$$
\frac{d \ln H}{d \ln a}+\frac{1}{2} \frac{d \ln \Omega_{\Lambda}}{d \ln a}=\frac{\sqrt{\Omega_{\Lambda}}}{c}-1 .
$$

In this manner we further obtain the evolution of $\Omega_{\Lambda}$ from the above Eqs. (8) and (22)

$$
\frac{d \Omega_{\Lambda}}{d z}=-2 \Omega_{\Lambda}\left(1-\Omega_{\Lambda}\right)\left(\frac{1}{2}+\frac{\sqrt{\Omega_{\Lambda}}}{c}\right)(1+z)^{-1} .
$$

Same as the other scenarios but for Eqs. (7) and (22), the EoS for HDE is found to be

$$
\omega_{\Lambda}=-\frac{1}{3}\left(\frac{2}{c} \sqrt{\Omega_{\Lambda}}+1\right) .
$$

Obviously, the acceleration condition $\omega<-1 / 3$ is satisfied as long as $c>0$. With $c \rightarrow \infty$, we have $\omega \rightarrow-1 / 3$. This is distinguished from the previous two scenarios. As with the other models, the Hubble expansion rate $E(z)$ follow easily.

\section{OBSERVATIONAL CONSTRAINTS AND THE ENERGY CONDITIONS}

Holographic and agegraphic dark energy models have been examined using various astronomical observations, such as 
SNIa [42] and CMB [43], among others. Indeed, constraint on HDE from OHD is also performed [37, 44]. Using the OHD and $\chi^{2}$ statistics, the authors gave the best-fit values of parameters $\Omega_{m 0}$ and $c$, suggesting that $c<1$ is favored. Various estimations of the $c$ parameter has been made [35, 42, 45], however there appears to be little consensus about its precise range. The constant $c$ is a key parameter in the HDE model which represents the proportion of dark energy component as shown in Eq. (1). In this paper, we therefore try to use the current more abundant OHD and SNIa compilation with the energy conditions for a further study. These two datasets are sensitive to the cosmic evolution in the dark energy-dominated era and turn out to form good complementary constraints.

\section{A. Hubble parameter}

Observational Hubble parameter data (OHD) generally can be measured through the differential ages of galaxies [46-50] and the baryon acoustic oscillation (BAO) peaks in the galaxy power spectrum [51]. They have been used in many cosmological models within the FRW framework [37, 48, 52, 53], and even the Lemaître-Tolman-Bondi (LTB) void models [54]. In this paper, we adopt the updated 28 OHD listed in the literature [50, 55]. It is worth mentioning that the latest measurement does not only extend to the deeper redshift realm, i.e., $H(z=2.3)=224 \pm 8 \mathrm{~km} \mathrm{~s}^{-1} \mathrm{Mpc}^{-1}$ [56], but also reveal a completely new method to obtain the OHD, namely, the BAO peak method using the Ly $\alpha$ forest.

For the OHD, parameters can be determined by following $\chi^{2}$ statistics

$$
\chi_{\mathrm{OHD}}^{2}\left(H_{0}, z, \mathbf{p}\right)=\sum_{i} \frac{\left[H_{0} E\left(z_{i}\right)-H^{o b s}\left(z_{i}\right)\right]^{2}}{\sigma_{i}^{2}},
$$

where $\mathbf{p}$ stands for the parameter vector of each dark energy models. In calculation, we adopt the latest measurement of $H_{0}$ by Planck [57] as the prior, $H_{0}=67.3 \pm 1.2 \mathrm{~km} \mathrm{~s}^{-1} \mathrm{Mpc}^{-1}$.

\section{B. Luminosity distance}

The SNIa sample is widely used for its famous richness. In this paper, we use the latest supernova Union2.1 compilation of 580 dataset [58]. The data are presented as tabulated distance moduli with errors. By definition, the theoretical distance modulus $\mu^{\text {th }}$ is the difference between the apparent magnitude $m$ and the absolute magnitude $M$

$$
\mu^{t h}(z)=m(z)-M=5 \log D_{L}(z)+\mu_{0},
$$

where $\mu_{0}=42.38-5 \log h$, and $h$ is the Hubble constant $H_{0}$ in units of $100 \mathrm{~km} \mathrm{~s}^{-1} \mathrm{Mpc}^{-1}$. The luminosity distance function $D_{L}(z)$ in a flat space can be expressed as

$$
D_{L}(z)=(1+z) \int_{0}^{z} \frac{d z^{\prime}}{E\left(z^{\prime} ; \mathbf{p}\right)}
$$

where the dimensionless Hubble expansion rate $E\left(z^{\prime} ; \mathbf{p}\right)$ is the connection between observation and model through Eq. (9).
Thus, the $\chi^{2}$ statistics can be constructed imitating the Eq. 25) but replacing Hubble parameter as distance modulus. However, in order to abandon the nuisance parameter $\mu_{0}$, an alternative way generally can be performed by marginalizing over it [59-61]. An new form of the $\chi^{2}$ statistics independent of $\mu_{0}$ is eventually reconstructed as

$$
\tilde{\chi}^{2}(z, \mathbf{p})=A-\frac{B^{2}}{C},
$$

where

$$
\begin{aligned}
A(\mathbf{p}) & =\sum_{i} \frac{\left[\mu^{o b s}(z)-\mu^{t h}\left(z ; \mu_{0}=0, \mathbf{p}\right)\right]^{2}}{\sigma_{i}^{2}(z)}, \\
B(\mathbf{p}) & =\sum_{i} \frac{\mu^{o b s}(z)-\mu^{t h}\left(z ; \mu_{0}=0, \mathbf{p}\right)}{\sigma_{i}^{2}(z)}, \\
C & =\sum_{i} \frac{1}{\sigma_{i}^{2}(z)} .
\end{aligned}
$$

This program has been widely used in the parameter constraint [62], reconstruction of the energy condition history [23] etc.

\section{Energy conditions}

By virtue of the Eqs. (1), (3) and (4), the energy conditions can be expressed in the below forms. The NEC suggests

$$
1+\omega \Omega_{\Lambda} \geqslant 0
$$

the WEC:

$$
3 M_{p}^{2} H^{2} \geqslant 0 \text { and } 1+\omega \Omega_{\Lambda} \geqslant 0
$$

the SEC:

$$
1+3 \omega \Omega_{\Lambda} \geqslant 0 \text { and } 1+\omega \Omega_{\Lambda} \geqslant 0
$$

the DEC:

$$
3 M_{p}^{2} H^{2} \geqslant 0,1+\omega \Omega_{\Lambda} \geqslant 0, \text { and } 1-\omega \Omega_{\Lambda} \geqslant 0 .
$$

Because of the non-negativity of $3 M_{p}^{2} H^{2}$, WEC eventually reduces to NEC.

Assuming the energy conditions, some bounds can be placed on parameters $\omega$ and $\Omega_{\Lambda}$. Furthermore, the EoS parameter $\omega$ can be constructed from parameters $\Omega_{\Lambda}$ (or $\Omega_{m}$ ) and $c$, which will be shown in next section. Thus, the energy conditions actually provide a series of constraints on parameters $\Omega_{m}$ and $c$. Note that the dark energy component $\Omega_{\Lambda}$ varies with redshift $z$, and its evolution has been shown in the above section. This means that we cannot expect the energy conditions to be constantly fulfilled or violated.

\section{Joint data constraints and energy condition bounds}

Having carried out the $\chi^{2}$ analysis with our three models, we are able to report the resulting constraint on the parameters, as shown by Figure 1 As can be seen from that figure, 

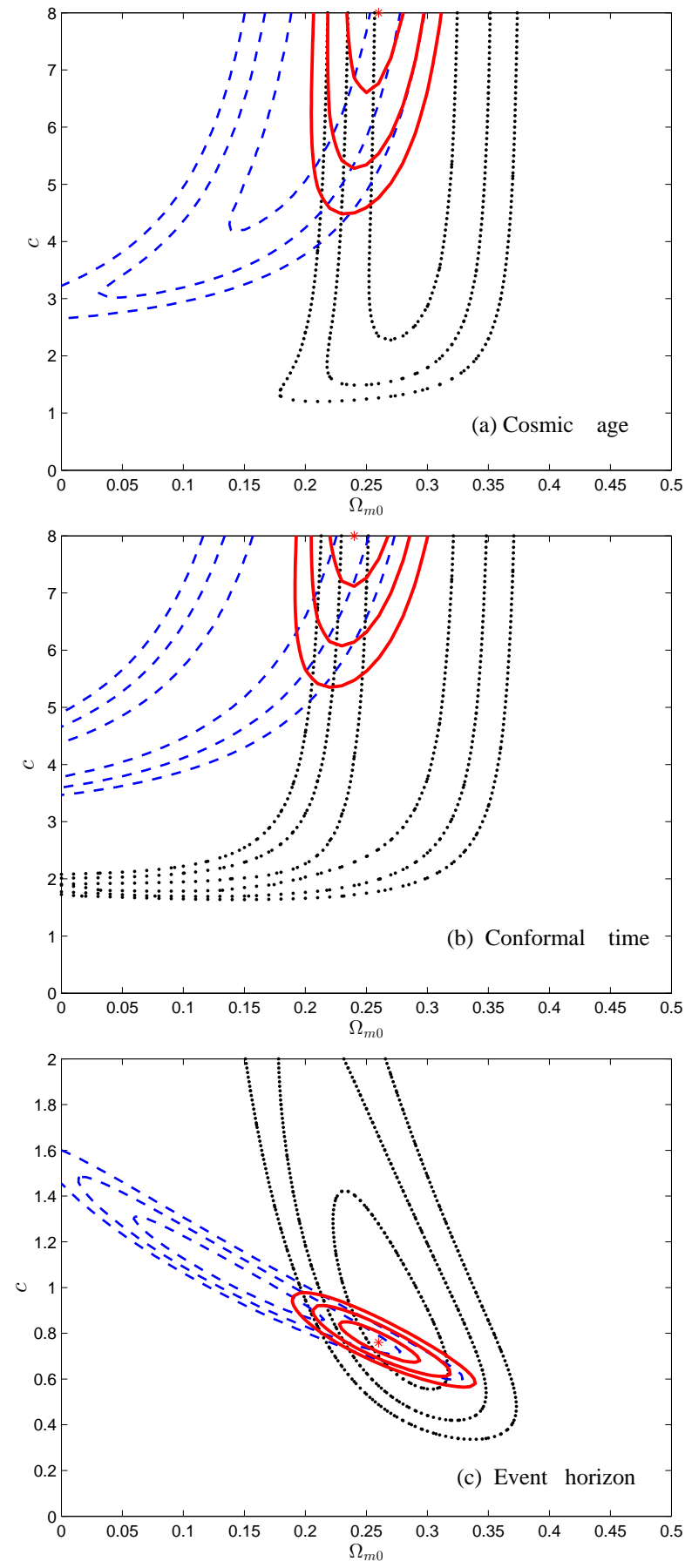

Figure 1: Constraints on parameters $\left(\Omega_{m 0}, c\right)$ from OHD and SNIa for the three models. The dashed and dotted contours are obtained from individual SNIa and OHD, respectively. The solid contours are joint constraints for OHD and SNIa. The contour levels correspond to $68.3 \%, 95.4 \%$ and $99.7 \%$ confidence regions, respectively. In the calculation of OHD, the latest measurement $H_{0}=67.3 \pm 1.2 \mathrm{~km} \mathrm{~s}^{-1}$ $\mathrm{Mpc}^{-1}$ [57] is used as the prior. The cut-off scenarios are indicated in the figure labels (a) to (c). the OHD and SNIa sets display fairly good complementarity, despite some trouble with bounding the $c$ parameter. Together they are able to reduce the parameter degeneracy and give us satisfactory bounds on $\Omega_{m 0}$ for all the three models. Even if they cannot always bound the parameter $c$, when used together the data improves our lower estimate of its possible range by a significant margin.

For the cosmic age cut-off model, we find $\Omega_{m 0}=0.26 \pm 0.02$ with $\chi_{\min }^{2}=592.25$. For a fair comparison between models, we also consider a conventional criterion in the literature, i.e., the minimum value in per degree of freedom $\chi_{\min }^{2} / d o f=0.977$. We know that the smaller $\chi_{\min }^{2} / d o f$ is a better choice for models. Assuming a prior on constant $0<c<8$, we find that the upper limit for $c$ is absent but $c>6.60$ at $68.3 \%$ confidence level. In fact, it is consistent with that of the interaction models [38]. We, therefore, could conclude that the spatial structure in form of cosmic age cut-off is the most essential-regardless of whether there exists interaction or not between interior matter. On the other hand, HDE finally can reduce to the standard cosmological model just because of the fundamental requirement $c \rightarrow \infty$ in this model. Using the fitted parameters, we estimate the EoS today of HDE at $\omega_{\Lambda}=-0.994$, very approach to the cosmological constant. For comparison, bounds required by the energy conditions at redshift $0 \leqslant z \leqslant 0.35$ are shown in Figure2. As for the energy conditions, we find that both NEC and DEC are trivially satisfied by the constrained regions for the entire redshift range. However, the data are in tension against SEC fulfillment at redshift $z=0$. In addition, we plot the evolution of the SEC bound at different redshift. We find that SEC is fulfilled at redshift $z \gtrsim 0.28$ with $1 \sigma$ confidence level, and $z \gtrsim 0.3$ with $3 \sigma$ confidence level. We also note that the observations indicate a violation of SEC at $z<0.19$.

For the conformal time cut-off model, constraints from data and the energy conditions are shown in Figure 3. The joint constraint for OHD and SNIa gives a little lower value of $\Omega_{m 0}=0.24_{-0.02}^{+0.03}$, a higher constant $c>7.11$ at $68.3 \%$ confidence level and a worse $\chi_{\min }^{2} / d o f=0.983$. EoS today of $\mathrm{HDE}$ in this model is also estimated at $\omega_{\Lambda}=-0.994$, very approach to the cosmological constant. NEC and DEC are almost kept stably consistently for the redshift range. This is same as the situation in age of universe cut-off. Moreover, an analogous result for SEC is obtained. That is, SEC access the valid region at $z \approx 0.2$. However, complete validity within $1 \sigma$ may occur at $z \approx 0.3$.

Finally we perform the $\chi^{2}$ analysis on the event horizon cut-off model. They present a moderate estimation for matter density $\Omega_{m 0}=0.26 \pm 0.04$ and a closed constraint on $c=0.76 \pm 0.06$. A similar evaluation as the cosmic age cutoff model is also available, i.e., $\chi_{\min }^{2}=592.77$ and $\chi_{\min }^{2} /$ dof $=$ 0.978 . Moreover, current equation of state of dark energy is estimated to be $\omega_{\Lambda}=-1.08 \pm 0.06$, a little deviation from the $\Lambda \mathrm{CDM}$ model. Comparison with energy condition bounds is shown in Figure 4. We note that NEC can give a much more meaningful bound for this scenario, especially at $z=0$. Moreover, we also note that it can directly and effectively compare with the constraint of OHD. For SEC, a familiar result is obtained, i. e. fulfill data at redshift $z \gtrsim 0.26$ with $1 \sigma$ confidence 


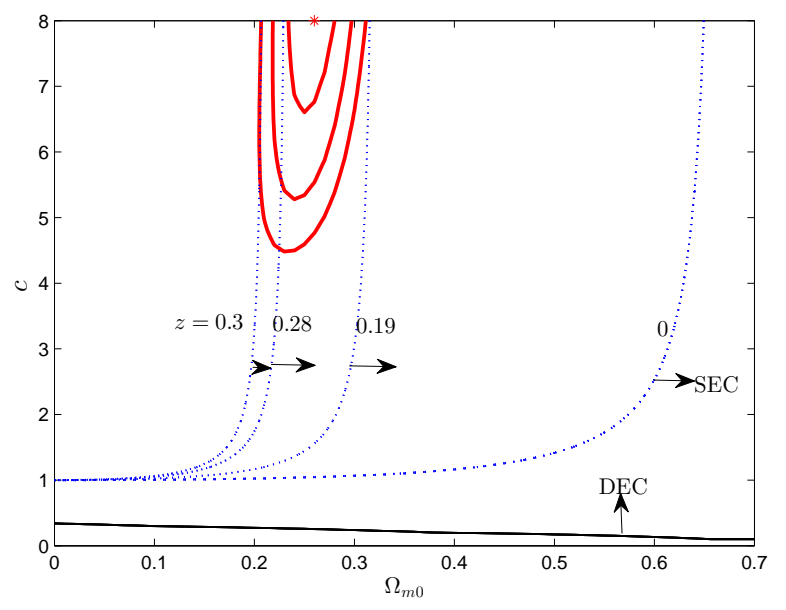

Figure 2: Observational constraints and the energy condition bounds for the cosmic age cut-off. The solid contours correspond to the joint constraint from OHD and SNIa data. Energy conditions bounds are labeled by redshift, and arrows point to the direction where the conditions are (or use to be) fulfilled. NEC bounds cover the whole parameter space, which is too trivial to be plotted. Constraint from SEC changes evidently with redshift, but constraint from DEC barely changes. Since constraints from the energy conditions are redshift dependent, i. e. constraints on $\Omega_{m}(z)$, we have transferred the unit into the current parameter space $\left(\Omega_{m 0}, c\right)$ according to the evolution of matter.

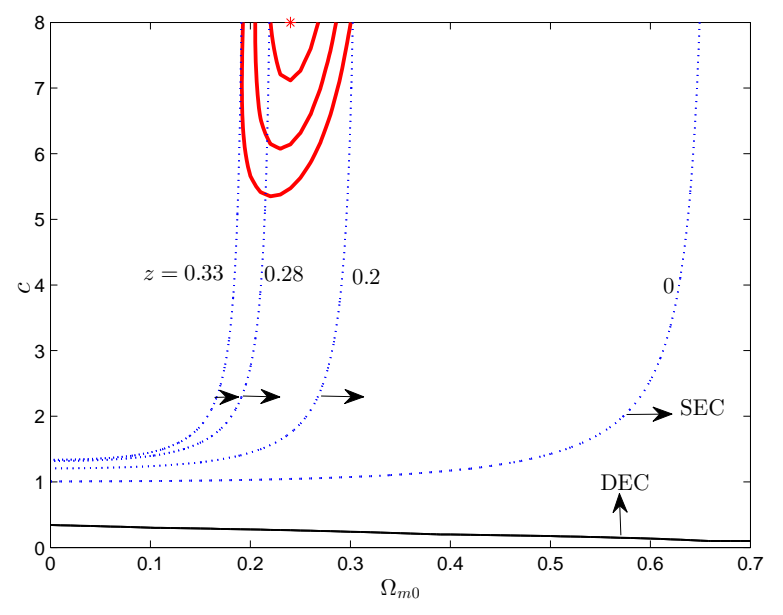

Figure 3: Same as Figure 2 but for the conformal time cut-off. Analogous results for SEC are obtained.

level, and $z \gtrsim 0.30$ with $3 \sigma$ confidence level. Most noteworthy is that the SEC from validity to complete validity within $3 \sigma$ only across the redshift span $0.17 \lesssim z \lesssim 0.30$, which can be used a good tool to test some models.

\section{CONCLUSION AND DISCUSSIONS}

We perform $\chi^{2}$ statistics to constrain the holographic and agegraphic dark energy models using latest OHD and SNIa Union2.1 compilation. The best-fit value of matter density

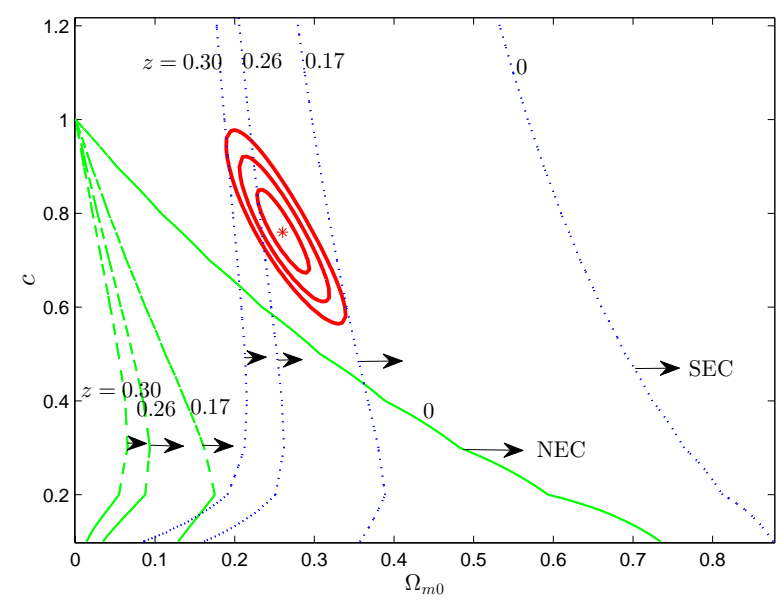

Figure 4: Same as Figure 2 but for the event horizon cut-off. Dashed curves show the boundaries of constraint from NEC.

for the three scenarios almost steadily located at $\Omega_{m 0}=0.26$ with $1 \sigma$ confidence level. The main results are shown in Figure 11 It turns out that $c$ cannot be constrained with a closed region for the age and conformal time cut-off, which is consistent with that of the interacting models [38]. We, therefore, could conclude that the spacetime structure in form of cosmic age cut-off is the most essential-regardless of whether there exists interaction or not between matter and dark energy. As discussed above, these two models eventually approaches the $\Lambda C D M$ model. The EoS today is estimated towards $\omega_{\Lambda}=-0.994$ for the two models. The constant $c$ is closed constrained at $c=0.76 \pm 0.06$ from joint data for the event horizon cut-off. The EoS in the event horizon cut-off is thus reconstructed as $\omega_{\Lambda}=-1.08 \pm 0.06$, a little deviation from the $\Lambda \mathrm{CDM}$ model. Furthermore, we consider the evaluation criterion $\chi_{\min }^{2} / d o f$ between models. We find that neither of this several models seem to work very well. By contrast, the model with conformal time cut-off is the worst.

The energy conditions turn out an useful approach to the study of cosmology, because of its model independence and universality. In our analysis, we superimpose the energy conditions bounds on the constraints from observational data as done in Figures 2, 3, and 4 at different redshift. In these scenarios, we find that SEC is fulfilled at redshift $0.2 \lesssim z \lesssim 0.3$ with $1 \sigma$ confidence level. The observations also require a validity of SEC with $3 \sigma$ confidence level at $z>0.3$. Moreover, the SEC is violated by the observations at $z \lesssim 0.2$. Theoretically, such a small error could provide a good constraint to future model examination. Moreover, constraint of the energy condition is physical and model-independent. Unfortunately, SEC is violated currently, which is expected in [23, 28]. Moreover, we find that NEC can provide an additional effective constraint on $\Omega_{m 0}$ and constant $c$ for the event horizon cut-off, when it is compared with OHD only. In fact, as far back as 1997, Visser [24] has inspected the observation in the epoch of galaxy formation using the energy condition, and predict an abnormal matter.

Compared with the work of $\mathrm{Wu}$ et al. [23], our results are 
obtained from a much narrower class of models. However, it should be noted that their work assumed a family of discontinuous functions as the bases on which the kinematic terms are expanded. Our result, in contrast, is not subjected to this limitation, but evolution of the energy condition. Therefore our analysis cannot be viewed as a special case of theirs. Importantly, our result exactly determine the fulfilment region of SEC, namely $0.2 \lesssim z \lesssim 0.3$ for $1 \sigma$ confidence level, and is less ambiguous at higher redshift.

It is possible, according to our results, to distinguish NEC as the energy condition primus inter pares. We find that, models that cannot be well-constrained by the data will also fail by NEC, yielding too generous a bound. For the model capable of being constrained by data, NEC gives a meaningful constraint that is neither in conflict with, nor entirely implied by the data. In future analysis of other dark energy proposals, therefore, NEC may be a worthwhile theoretical consideration before the application of data analysis.

However, we have to admit a fact that only OHD and SNIa compilations are used in present paper, although they are the latest ones. If more sufficient data were considered, such as WMAP, BAO etc., the constraint would be tighter and stronger. And power of the energy conditions would also be disabled. Repeated again, we have considered the HDE mod- els without interaction according to a fact [33]. However, we should also note an important phenomenon. For the interacting models, degeneracy between matter density and constant $c$ shown in Figures 2, 3 and 4 of Chen et al. [38] is different from the one of this paper. From the opposing direction of contour constraints, we have the possibility to expect that the energy condition could play an important role in the interacting models. What we can not ignore is that neither the $\chi_{\min }^{2} / d o f$ nor the AIC and BIC criteria [37] under current observations rule out some of these models unambiguously. It is, therefore, reasonable to expect a powerful future observation to distinguish them.

\section{Acknowledgments}

We thank the anonymous referee whose suggestions greatly helped us improve this paper. This work was supported by the National Science Foundation of China (grant No. 11173006), the Ministry of Science and Technology National Basic Science program (project 973) under grant No. 2012CB821804, and the Fundamental Research Funds for the Central Universities.
[1] A. Riess et al., Astron. J 116, 1009 (1998).

[2] M. Tegmark, M. Strauss, M. Blanton, et al., Physical Review D 69, 103501 (2004).

[3] D. Spergel et al., Astrophys. J. Suppl 148, 170 (2003).

[4] P. Peebles and B. Ratra, The Astrophysical Journal 325, L17 (1988).

[5] C. Armendariz-Picon, V. Mukhanov, and P. Steinhardt, Physical Review Letters 85, 4438 (2000).

[6] T. Padmanabhan, Physical Review D 66, 021301 (2002).

[7] R. Caldwell, M. Kamionkowski, and N. Weinberg, Physical Review letters 91, 71301 (2003).

[8] F. Piazza and S. Tsujikawa, Journal of Cosmology and Astroparticle Physics 2004, 004 (2004).

[9] B. Feng, X. Wang, and X. Zhang, Phys. Lett., B 607, 35 (2005).

[10] M. Özer and M. Taha, Physics Letters B 171, 363 (1986).

[11] V. Sahni and A. Starobinsky, International Journal of Modern Physics D 9, 373 (2000).

[12] L. Susskind, Journal of Mathematical Physics 36, 6377 (1995), arXiv:hep-th/9409089.

[13] G. t Hooft, Basics and Highlights in Fundamental Physics 1, 72 (2001).

[14] A. Cohen, D. Kaplan, and A. Nelson, Physical review letters 82, 4971 (1999).

[15] P. Hořava and D. Minic, Physical Review Letters 85, 1610 (2000).

[16] S. Thomas, Physical Review Letters 89, 081301 (2002).

[17] S. D. Hsu, Physics Letters B 594, 13 (2004).

[18] K. Y. Kim, H. W. Lee, and Y. S. Myung, Physics Letters B 660, 118 (2008).

[19] H. Wei, Nuclear Physics B 819, 210 (2009).

[20] S. Hawking and G. Ellis, The large scale structure of spacetime, vol. 1 (Cambridge Univ Pr, 1975).

[21] R. Wald, General relativity (University of Chicago press, 1984).
[22] S. Carroll, Spacetime and geometry (Addison-Wesley, Pearson, 2004).

[23] C. Wu, C. Ma, and T. Zhang, The Astrophysical Journal 753, 97 (2012).

[24] M. Visser, Science 276, 88 (1997).

[25] M. Visser, Physical Review D 56, 7578 (1997).

[26] M. Visser and C. Barcelo, Cosmo99 Proceedings pp. 98-112 (2000).

[27] C. Cattoën and M. Visser, Classical and Quantum Gravity 25, 165013 (2008).

[28] M. Lima, S. Vitenti, and M. Rebouças, Physical Review D 77, 083518 (2008).

[29] M. Li, X. Li, S. Wang, and Y. Wang, Communications in Theoretical Physics 56, 525 (2011).

[30] P. Schuecker, R. Caldwell, H. Böhringer, C. Collins, L. Guzzo, and N. Weinberg, Astronomy and Astrophysics 402, 53 (2003).

[31] H. Epstein, V. Glaser, and A. Jaffe, Nuovo Cimento 36, 1016 (1965).

[32] C. J. Fewster and S. P. Eveson, Physical Review D 58, 084010 (1998).

[33] Q. Wu, Y. Gong, A. Wang, and J. Alcaniz, Physics Letters B 659, 34 (2008).

[34] M. Li, Physics Letters B 603, 1 (2004).

[35] X. Zhang and F.-Q. Wu, Physical Review D 76, 023502 (2007).

[36] M. Setare, Physics Letters B 642, 1 (2006).

[37] Z.-X. Zhai, T.-J. Zhang, and W.-B. Liu, Journal of Cosmology and Astroparticle Physics 2011, 019 (2011).

[38] Y. Chen, Z. Zhu, L. Xu, and J. Alcaniz, Physics Letters B 698, 175 (2011).

[39] L. Xu, J. Lu, and W. Li, Physics Letters B 690, 333 (2010).

[40] Y. Myung and M. Seo, Physics Letters B 671, 435 (2009).

[41] S. Bhattacharya and U. Debnath, International Journal of Modern Physics D 20, 1191 (2011). 
[42] Q. Huang and Y. Gong, Journal of Cosmology and Astroparticle Physics 08, 006 (2004).

[43] K. Enqvist, S. Hannestad, and M. Sloth, Journal of Cosmology and Astroparticle Physics 02, 004 (2005).

[44] Z.-L. Yi and T.-J. Zhang, Modern Physics Letters A 22, 41 (2007).

[45] H.-C. Kao, W.-L. Lee, and F.-L. Lin, Physical Review D 71, 123518 (2005).

[46] R. Jimenez and A. Loeb, The Astrophysical Journal 573, 37 (2002).

[47] J. Simon, L. Verde, and R. Jimenez, Physical Review D 71, 123001 (2005).

[48] D. Stern, R. Jimenez, L. Verde, M. Kamionkowski, and S. Stanford, Journal of Cosmology and Astroparticle Physics 2010, 008 (2010).

[49] M. Moresco, A. Cimatti, R. Jimenez, L. Pozzetti, G. Zamorani, M. Bolzonella, J. Dunlop, F. Lamareille, M. Mignoli, H. Pearce, et al., Journal of Cosmology and Astroparticle Physics 2012, 006 (2012).

[50] C. Zhang, H. Zhang, S. Yuan, T.-J. Zhang, and Y.-C. Sun, arXiv preprint arXiv:1207.4541 (2012).

[51] E. Gaztanaga, A. Cabré, and L. Hui, Monthly Notices of the Royal Astronomical Society 399, 1663 (2009).

[52] L. Samushia and B. Ratra, The Astrophysical Journal Letters 650, L5 (2006).
[53] T.-J. Zhang, C. Ma, and T. Lan, Advances in Astronomy 2010, 81 (2010).

[54] H. Wang and T.-J. Zhang, The Astrophysical Journal 748, 111 (2012).

[55] O. Farooq and B. Ratra, The Astrophysical Journal Letters 766, L7 (2013).

[56] N. G. Busca, T. Delubac, J. Rich, S. Bailey, A. FontRibera, D. Kirkby, J.-M. Le Goff, M. M. Pieri, A. Slosar, É. Aubourg, et al., Astronomy \& Astrophysics 552, A96 (2013), arXiv:1211.2616.

[57] P. Collaboration, P. Ade, N. Aghanim, C. Armitage-Caplan, M. Arnaud, M. Ashdown, F. Atrio-Barandela, J. Aumont, C. Baccigalupi, A. Banday, et al., arXiv preprint arXiv:1303.5076 (2013).

[58] N. Suzuki, D. Rubin, C. Lidman, G. Aldering, R. Amanullah, K. Barbary, L. Barrientos, J. Botyanszki, M. Brodwin, N. Connolly, et al., The Astrophysical Journal 746, 85 (2012).

[59] E. D. Pietro and J.-F. Claeskens, Monthly Notices of the Royal Astronomical Society 341, 1299 (2003).

[60] S. Nesseris and L. Perivolaropoulos, Physical Review D 72, 123519 (2005).

[61] L. Perivolaropoulos, Physical Review D 71, 063503 (2005).

[62] H. Wei, Journal of Cosmology and Astroparticle Physics 2010, 020 (2010). 